\title{
Análise da Degradação Ambiental na Amazônia Ocidental: um Estudo de Caso dos Municípios do Acre ${ }^{1}$
}

\author{
Rubicleis Gomes da Silva ${ }^{2}$ \\ Claudiney Guimarães Ribeiro ${ }^{3}$
}

Resumo - Os resultados gerais divulgados pelo relatório final do GOVERNO DO ESTADO DO ACRE (2000) mostraram que parte da região sudeste do Estado do Acre vem sendo modificada há algumas décadas por um processo de ocupação iniciado por desflorestamentos e atividades agropecuárias. Segundo este relatório, o desmatamento e a queima da vegetação por atividades humanas foram as grandes transformadoras das paisagens acreanas e continuam a crescer muito nas últimas décadas. Diante do exposto, o objetivo principal deste trabalho foi elaborar um Índice de Degradação (ID) que fosse capaz de medir o atual estágio de degradação ambiental em que se encontram os 22 municípios do Estado do Acre, na região norte do Brasil, procurando, também, agrupálos em clusters para verificar características semelhantes entre eles. Os resultados indicam que o Estado do Acre possui um ID médio de 30,74\%, possuindo como ID’s mínimos os municípios de Cruzeiro do Sul e

\footnotetext{
${ }^{1}$ Os autores agradecem a CAPES e a Universidade Federal do Acre pelo financiamento da pesquisa e aos professores J oão Eustáquio de Lima e José Euclídes A. Cavalcanti pelas críticas e sugestões.

2 Economista, servidor da Universidade Federal do Acre (UFAC), Mestre em Economia Aplicada e Doutorando em Economia pelo Departamento de Economia Rural (DER) da Universidade Federal de Viçosa (UFV). E-mail: rubicleis@uol.com.br

${ }^{3}$ Economista, professor de economia da Universidade Federal de São João Del Rei e Mestre em Economia Aplicada pelo Departamento de Economia Rural (DER) da Universidade Federal de Viçosa (UFV). E-mail: cgribeiro_br@yahoo.com.br
} 
Mâncio Lima com ID igual a zero e Manoel Urbano com um ID de $1,80 \%$. A análise de cluster mostrou que determinadas regiões possuem um bom estado de conservação ambiental, no entanto, também deixou claro que alguns indicadores de degradação apresentam valores extremamente elevados.

Palavras Chaves: Índice de Degradação, indicadores ambientais, estado do Acre.

Abstract - The general results disclosed by the State of Acre's final report (2000) showed that part of the Southeast area of the State has been modified for some decades by an occupation process triggered by deforestation and agricultural and cattle raising activities. According to this report, deforestation and burning of vegetation by human activities were the major transformers of the State landscapes and have increased in the last decades. Therefore, the main purpose of this work was to develop a Degradation Index (DI) that would measure the current stage of environmental degradation of the 22 municipalities of State of Acre in the North region of Brazil also attempting to group them in clusters to verify similar characteristics among them. The results indicate that the State of Acre has an average DI of $30,74 \%$, with minimum DI's in the municipalities of Cruzeiro do Sul and Mâncio Lima with DI equal to zero and Manoel Urbano with a DI equal to $1,80 \%$. The cluster analysis showed that certain regions present a good state of environmental conservation, but also made clear that some degradation indicators present extremely high values.

Key words: Degradation Index, environmental indicators, State of Acre.

\section{1 - Introdução}

Ao longo de sua história, a ocupação do território e a organização de atividades econômicas no Acre, respaldados por políticas e projetos governamentais, tipicamente beneficiaram determinados grupos da 
população no curto prazo, sem viabilizar um modelo de desenvolvimento duradouro, com benefícios para todos a médio e longo prazos (GOVERNO DO ESTADO DO ACRE, 2000).

A partir dos anos 70, a expansão da fronteira agropecuária e madeireira (ainda que de forma menos intensa em relação a outros estados como Pará, Mato Grosso e Rondônia) foi acompanhada por uma série de problemas graves dentre os quais pode-se destacar os conflitos sociais quanto ao acesso à terra e a outros recursos naturais, exploração insustentável de recursos naturais, altas taxas de desistência nos projetos de assentamento, crescimento desordenado de cidades como Rio Branco, entre outros. (GOVERNO DO ESTADO DO ACRE, 2000).

Em grande medida, esses problemas resultaram da falta de incorporação, dentro das políticas e projetos governamentais, dos princípios básicos do Desenvolvimento Sustentável. Buscando reverter esta situação, o governo do Estado do Acre elaborou recentemente 0 Zoneamento Ecológico-Econômico (ZEE), tendo por objetivo principal contribuir para a incorporação dos princípios de Desenvolvimento Sustentável na orientação das ações do governo, do setor privado e da sociedade em geral. (GOVERNO DO ESTADO DO ACRE, 2000).

Os resultados gerais divulgados pelo relatório final do GOVERNO DO ESTADO DO ACRE (2000) mostraram que parte da região sudeste do Estado vem sendo modificada há algumas décadas, com variados graus de intensidade, por processos de ocupação iniciados por desmatamentos extensos e atividades agropecuárias, sendo que o restante do Estado encontra-se em níveis diferenciados de alteração.

Segundo este relatório a derrubada da floresta e a queima da vegetação por atividades humanas foram as grandes transformadoras das paisagens acreanas e continuam a crescer muito nas últimas décadas. Resultados do levantamento feito para os temas "desflorestamentos e queimadas" mostraram que: i) o desflorestamento vem aumentando no Estado, a uma taxa média de 0,35\% a.a. $(53.349,26 \mathrm{ha} /$ ano), sendo que no período de 1994/95 esta taxa chegou a 0,86\% $(131.086,77)$; ii) as regionais do Alto e Baixo Acre foram as que apresentaram os maiores índices de desflorestamentos e queimadas; e, iii) a existência de uma alta correlação entre a expansão da pecuária e o desflorestamento. 
Diante do exposto, o objetivo principal deste trabalho foi elaborar um Índice de Degradação (ID) que fosse capaz de aferir o atual estágio de degradação ambiental em que se encontram os 22 municípios do Estado do Acre, na região norte do Brasil, procurando, também, agrupálos em clusters para verificar características semelhantes entre eles. Adicionalmente, procurou-se confrontar os resultados obtidos na presente pesquisa com os resultados publicados no relatório final do GOVERNO DO ESTADO DO ACRE (2000), buscando compatibilizá-los, verificando o grau de coerência existente entre eles.

\section{2- Metodologia}

O Índice de Degradação (ID), mede a proporção de degradação da área de determinado município. Este índice tem como referência o trabalho de LEMOS (2000) e constitui-se, segundo este autor, numa evolução, do ponto de vista metodológico, do Índice de Desertificação por ele desenvolvido. Segundo LEMOS (1995), o Índice de Desertificação não captava o percentual de devastação a que cada um dos municípios de seu campo de estudo (região nordeste) estava sendo submetido, o que se torna possível a partir da construção do ID. Neste sentido, a construção do ID foi feita em duas etapas. Na primeira, desenvolveu-se, por meio de procedimento de análise multivariada, o Índice Parcial de Degradação (IPD). O software utilizado foi o SPSS 10.0, procedimento Data Reduction - Factor. Com base no IPD foram estimados, utilizando-se análise de regressão, os pesos atribuídos a cada uma das variáveis que entraram na composição do ID. O software utilizado foi o EViews 4.0, método dos mínimos quadrados ordinários (MQO).

\section{1 - Estimação do Índice Parcial de Degradação (IPD)}

Para estimar o IPD associado a cada um dos municípios acreanos, utilizou-se um dos procedimentos existentes de análise multivariada. Mais especificamente, empregou-se o método de análise fatorial com decomposição em Componentes Principais. O referido método é apresentado a seguir em sua forma matricial. 


\subsection{1 - O modelo de Análise Fatorial}

Genericamente, um modelo de análise fatorial é apresentado da seguinte forma:

$$
X=\mu+\mathrm{a} f+\mathrm{e}
$$

em que $X=\left(X_{1}, X_{2}, \ldots, X_{p}\right)^{t}$ é um vetor transposto de variáveis aleatórias observáveis; $f=\left(f_{1}, f_{2}, \ldots, f_{r}\right)^{t}$ é um vetor transposto $(r<p)$ de variáveis não observáveis ou fatores; $\alpha$ é uma matriz ( $p \times r$ ) de coeficientes fixos denominados cargas fatoriais; $\mathrm{e}=\left(\mathrm{e}_{1}, \mathrm{e}{ }_{2}, \ldots, \mathrm{e}_{p}\right)^{t}$ é um vetor transposto de erros aleatórios.

Algumas propriedades do método podem ser destacadas. Uma delas é que $E(e)=E(f)=0$. Outra propriedade adicional associada aos fatores é que eles são ortogonais. Segundo LEMOS (2000), em geral a estrutura inicial das estimativas das cargas fatoriais não é definitiva. Para confirmar ou rejeitar esta estrutura, o método proporciona a possibilidade de se fazer sua rotação, sendo utilizado neste trabalho o método Varimax de rotação ortogonal dos fatores 4 .

Para a construção do IPD, estimaram-se os escores associados aos fatores obtidos após a rotação ortogonal da estrutura fatorial inicial (LEMOS, 2000). Por definição, o escore fatorial irá situar cada observação no espaço dos fatores comuns. Deste modo, para cada fator $\mathrm{f}_{\mathrm{i}} \mathrm{o} \mathrm{i}$ ésimo escore fatorial que pode ser extraído é definido por $F_{i}$ e pode ser expresso por:

$$
F_{i}=\sum_{i=1}^{n} b_{j} X_{i j}, \quad \operatorname{com} j=1,2, \ldots, p
$$

em que $b_{j}$ são os coeficientes de regressão e $X_{i j}$ são as $p$ variáveis observáveis.

Como a variável $F_{i}$ é não observável, tem-se que estimá-la através das técnicas de análise fatorial por meio da matriz $X$ de variáveis observáveis. Neste sentido, utilizando-se a forma matricial, pode-se reescrever a equação (2) da seguinte forma: 


$$
F_{(n \times q)}=X_{(n \times p)} \cdot B_{(p \times q)}
$$

Como nas equações (2) e (3) os escores fatoriais serão afetados tanto pela magnitude quanto pelas unidades em que as variáveis $X_{i}$ são medidas, substitui-se a variável $X_{i}$ pela variável normalizada $Z_{i j}$, podendo-se reescrever a equação (3) como segue:

$$
Z_{i j}=\left[\left(X_{i}-\mu_{x i}\right) / s_{x i}\right]
$$

em que $\mu_{\mathrm{xi}}$ é a média de $\mathrm{X}_{\mathrm{i}}$ e $\sigma_{\mathrm{xi}}$ é o seu desvio padrão.

A partir desta transformação, a equação (3) é modificada podendo ser reescrita da seguinte maneira:

$$
F_{(n \times q)}=Z_{(n \times p)} \cdot b_{(p \times q)}
$$

$\mathrm{Na}$ equação (4), o vetor $\beta$ substitui o vetor dos coeficientes de regressão $B$, pois as variáveis estão normalizadas em ambos os lados da equação. Ao se multiplicar os dois lados da equação (4) por (1/n) Zt, tem-se:

$$
(1 / n) Z^{t} F=(1 / n) Z^{t} Z B
$$

em que $n$ é o número de observações e $Z^{\mathrm{t}}$ é a matriz transposta de $Z$.

$O$ primeiro membro da equação $(5),(1 / n) Z^{t} F$, é a matriz de correlação entre os termos de $X_{i}$ e a partir de agora será representada por R. Já a matriz $(1 / n) Z Z Z \beta$ representa a correlação existente entre os escores fatoriais e os próprios fatores e será identificada por $\Lambda$. Assim, pode-se reescrever a equação (5) da seguinte forma:

$$
\Lambda=R \cdot \beta
$$

Supondo que a matriz $R$ é não-singular, ou seja, que $|R| \neq 0$, podese multiplicar ambos os lados de (6) pela inversa de $R$, dada por $\left(R^{-1}\right)$, obtendo-se a seguinte equação:

$$
\beta=R^{-1} . \Lambda
$$


Tendo-se estimado o vetor $\beta$, pode-se substituí-lo na equação (4), obtendo-se os escores fatoriais associados a cada observação.

\section{2 - Construção do IPD}

Para a elaboração do IPD, utilizou-se da propriedade de ortogonalidade dos escores fatoriais estimados, destacando-se que a ortogonalidade associada à matriz de fatores não implica necessariamente na ortogonalidade dos escores fatoriais. Portanto, deve-se testar se os escores fatoriais são ortogonais, observando-se a matriz de variância e covariância entre estes escores, a qual deve ser uma identidade para que os escores fatoriais sejam ortogonais (LEM OS, 2000).

O IPD foi estimado pela seguinte equação:

$$
\operatorname{IPD}_{i}=\left(\sum_{i=1}^{n} F_{i j}^{2}\right)^{\frac{1}{2}}, \operatorname{com} j=1,2, \ldots, p
$$

em que IPD é o índice parcial de degradação associado ao i-ésimo município do Acre; $F_{i j}$ são os escores fatoriais estimados segundo o procedimento de decomposição em componentes principais.

De acordo com LEMOS (2000), como se espera que os escores associados aos municípios tenham distribuição simétrica em torno da média zero, metade deles terá sinais negativos e a outra metade terá sinais positivos, sendo que os municípios que apresentarem os menores índices de degradação parcial terão escores fatoriais negativos. Para evitar que altos escores fatoriais negativos elevem a magnitude dos índices associados a estes municípios, torna-se necessária a transformação mostrada abaixo, tendo por objetivo inserí-los todos no primeiro quadrante:

$$
F_{i j}=\frac{\left(F-F_{\min }\right)}{\left(F_{\max }-F_{\min }\right)}
$$

em que $F_{\min }$ e $F_{\text {max }}$ são os valores máximo e mínimo observados para os escores fatoriais associados aos municípios acreanos. 
Com este procedimento todos os escores fatoriais estarão contidos no intervalo fechado entre zero e um. Na Figura 1 mostra-se como se calcula geometricamente o Índice Parcial de Degradação.

Figura 1 - Construção Geométrica do Índice Parcial de Degradação (IPD).

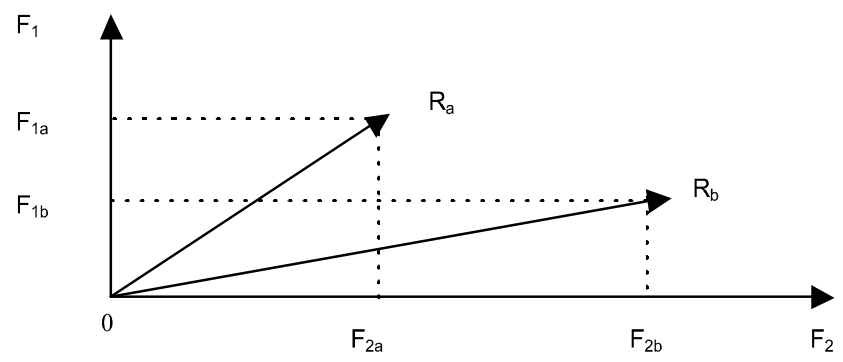

Por esta figura observa-se que associado ao município A estão os escores fatoriais $F_{1 a}$ e $F_{2 a}$. Segundo LEMOS (2000), a resultante associada a estes escores fatoriais ortogonais é dada pelo vetor $\mathrm{R}_{\mathrm{a}}$ assim definido:

$$
R_{a}=\left(F_{1 a}^{2}+F_{2 a}^{2}\right)^{1 / 2}
$$

O mesmo procedimento seria utilizado para o município $B$. A magnitude do IPD associado aos municípios A e B será obtida exatamente pelo tamanho da resultante $R_{a}$ ou $R_{b}$, respectivamente. Vale ressaltar que o IPD, definido desta forma, é de utilidade para fazer o rank dos municípios do Acre quanto ao nível de degradação (LEMOS, 2000). Não serve porém para estimar o percentual de degradação que é observado em cada um dos municípios, o que é feito utilizando-se o ID cuja formulação será apresentada a seguir.

\section{3 - Construção do ID}

Para construir o ID associado ao i-ésimo município acreano, utilizou-se a seguinte equação:

$$
I D_{i}=\left(\sum_{i=1}^{n} P_{j} X_{i}\right), \text { com } \sum_{j=1}^{p} P_{j}=1
$$


em que os pesos $P_{j}$ são estimados por regressão múltipla, onde a variável dependente é o $I P D_{i}$ e as variáveis explicativas são os indicadores utilizados para a construção do ID.

\subsection{1 - Construção dos Indicadores de Degradação}

No presente trabalho o termo degradação ambiental pode ser entendido como sendo os danos gerados ao meio ambiente por atividades econômicas, aspectos populacionais e fatores biológicos, ou seja, a degradação ambiental pode ser considerada como um processo caracterizado por desmatamentos para atender a demanda da população para novas áreas onde possam fixar-se, a derrubada da floresta e a queima da vegetação tendo por objetivo aumentar as áreas limpas para atender atividades econômicas como agricultura e pecuária. Neste sentido, para construir o ID, levou-se em consideração quatro indicadores, sendo um biológico, dois econômicos e um demográfico.

0 indicador biológico refere-se à cobertura vegetal existente em cada município do Acre. Esta cobertura é avaliada pelo somatório das áreas com matas e florestas nativas e plantadas, áreas com lavouras perenes e áreas com lavouras temporárias, sendo dividido pela área rural total do município (LEMOS, 2000).

Os dois indicadores econômicos foram definidos pela produtividade das lavouras e animal. 0 primeiro foi obtido pela relação entre 0 valor da produção vegetal agregada do município e a soma das áreas com lavouras perenes e temporárias. 0 segundo foi obtido pela divisão do valor da produção animal do município pela área total com pastagens naturais e cultivadas (LEM OS, 2000).

0 indicador demográfico refere-se à capacidade das áreas com lavouras (perenes e temporárias) e de pastagens (naturais e plantadas) suportarem um maior contingente de trabalhadores nas atividades agropecuárias. Quanto mais degradadas estiverem as áreas, menores serão as capacidades dessas áreas em suportar uma maior quantidade de trabalhadores por unidade. Portanto, esta variável se define pela relação entre a mão de obra total utilizada na zona rural do município dividida pelo somatório das áreas com lavouras e pastagens do município (LEMOS, 2000).

Segundo LEMOS (2000), para se construir um Índice de Degrada- 
ção seria necessário ter uma informação à priori de quais seriam os níveis ideais de preservação associados aos indicadores que são utilizados para a sua construção. Esta seria uma tarefa extremamente difícil, até porque variaria de acordo com quem estivesse fazendo a análise e dependeria de um forte nível de subjetividade.

Para contornar esta situação, foi adotado o critério de hierarquização dos 11 municípios melhores posicionados em cada um dos indicadores utilizados para aferir a degradação. Com base nestes 11 municípios, estimou-se uma média aritmética de cada indicador, tomando-se estes valores como referência de preservação. Logo, quanto mais distante estiver o valor encontrado de um município qualquer para um determinado indicador em relação à média estimada daquele indicador nos 11 municípios melhores posicionados, mais degradado estará 0 município no que se refere a este indicador específico (LEMOS, 2000).

Com base na descrição acima, definem-se os seguintes indicadores: - $\mathbf{C O B V}_{\mathbf{i}}=$ cobertura vegetal do município, que representa o somatório das áreas com matas e florestas nativas e cultivadas, mais as áreas com lavouras perenes e temporárias dividida pela área total do i-ésimo município do estado do Acre;

- COBV $_{\text {ReF }}=$ média da cobertura vegetal dos 11 municípios melhores posicionados em relação a este indicador;

- $\mathbf{V A V E}_{\mathbf{i}}=$ valor da produção vegetal do i-ésimo município do estado do Acre dividida pela soma das áreas com lavouras perenes e temporárias;

- VAVE $\mathbf{R E F}_{\mathbf{R E F}}=$ média deste indicador nos 11 municípios melhores posicionados em relação a ele;

- VANI = valor da produção animal do i-ésimo município do estado do Acre dividida pela área total com pastagens naturais e cultivadas;

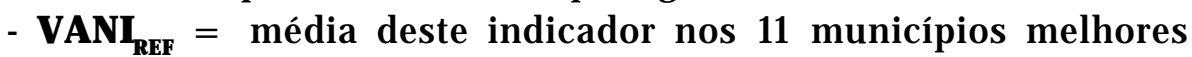
posicionados em relação a ele;

- $\mathbf{M O R U}_{\mathbf{i}}=$ total da mão de obra empregada no meio rural do município i-ésimo dividida pelo somatório das áreas com lavouras e pastagens;

- MORU $_{\mathbf{R E F}}=$ média deste indicador nos 11 municípios melhores posicionados em relação a ele.

A partir dos indicadores acima, desenham-se aqueles que entram na construção do IPD e do ID, cujas definições são as seguintes (LEMOS, 2000): 
- $\operatorname{DECOBV}\left(\mathrm{X}_{\mathrm{i1}}\right)=0$, quando COBV $\geq \mathrm{COBV}_{\mathrm{REF}} ;$

- DECOBV $\left(X_{i 1}\right)=\left[1-\left(\operatorname{COBV} / \operatorname{COBV}_{\mathrm{REF}}\right)\right] * 100$, nos demais casos;

- DEVAVE $\left(X_{i 2}\right)=0$, quando VAVE $\geq \operatorname{VAVE}_{\text {REF }} ;$

- DEVAVE $\left(X_{\mathrm{i} 2}\right)=\left[1-\left(\operatorname{VAVE} / \operatorname{VAVE}_{\mathrm{REF}}\right)\right]^{*} 100$, nos demais casos;

- DEVANI $\left(X_{i 3}\right)=0$, quando VANI $\geq$ VANI $_{\text {REF }} ;$

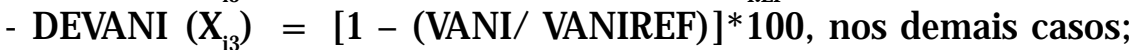

- DEMORU $\left(X_{i 4}\right)=0$, quando PORU $\geq$ PORU $_{\text {REF }} ;$

- DEMORU $\left(X_{i 4}\right)=\left[1-\left(P O R U / P O R U_{\text {REF }}\right)\right]^{*} 100$, nos demais casos.

Desta forma, tanto o IPD como o ID constituem-se em índices relativos de degradação, que têm como referência os resultados observados nos 11 municípios melhores posicionados em cada indicador que entra na sua composição.

Denominando a variável dependente IPD de $Y$ e os indicadores de degradação DECOBV, DEVAVE, DEVANI e DEMORU de $X_{1}, X_{2}, X_{3}$ e $X_{4}$ respectivamente, as condições de estabilidade do modelo são dadas por:

$$
\frac{\partial Y}{\partial X_{1}}>0, \frac{\partial Y}{\partial X_{2}}>0, \frac{\partial Y}{\partial X_{3}}>0 \text { e } \frac{\partial Y}{\partial X_{4}}>0
$$

Os dados utilizados foram obtidos no Censo Agropecuário de 1995/ 1996.

\section{4 - Método de Agrupamento}

\subsection{1 - Análise de Cluster}

De acordo com FERNAU e SAMSON (1990), a análise de agrupamento compõe-se de um conjunto de técnicas estatísticas cujo propósito é classificar os dados, unindo-os pelas semelhanças ou pelas diferenças, conforme o estudo. Os elementos de um mesmo grupo devem ser o mais semelhante possível entre si, enquanto a diferença entre os grupos deve ser a maior possível. A distância entre pontos é usualmente determinada pela distância euclidiana ou pelo coeficiente de correlação, podendo variar de 0 (variáveis idênticas) a $+\infty$ (variáveis sem relação) (GONG e RICHMAN, 1995).

Como destaca FERNANDES e LIMA (1991), se n medidas, para um 
elemento i, são dadas em um vetor $X_{i}(n \times 1)$, a distância do elemento i a outro elemento j pode ser calculada, na forma mais simples, pela seguinte expressão:

$$
D_{i j}=\left[\left(X_{i}-X_{j}\right)^{\prime}\left(X_{i}-X_{j}\right)\right]^{1 / 2}
$$

Ainda segundo FERNANDES e LIMA (1991), este cálculo pode ser generalizado para todos os elementos do estudo, sendo as distâncias representadas na matriz de proximidade e a anotação ij. A posição, nessa matriz, indica a distância entre o elemento $i$ e o elemento $j$.

Os métodos mais comuns de agrupamento para determinar a distância entre agrupamentos são: ligação simples, ligação completa e ligação pela média, centróide e método das médias, "Ward's" ou método da mínima variância (FERNAU e SAMSON, 1990; GONG e RICHMAN, 1995).

A análise de agrupamento envolve algumas decisões subjetivas, como qual a técnica constitui-se a mais conveniente, conforme as circunstâncias, quais as distâncias a serem consideradas, o número ótimo de agrupamentos, entre outras (FERNAU e SAMSON, 1990; POLLAK e CORBETT, 1993). Assim, dos métodos testados neste trabalho, o que apresentou resultado mais coerente ${ }^{5}$ foi o método de ligação simples (single linkage), baseado na distância euclidiana.

\section{3 - Resultados e Discussões}

Realizados os procedimentos de análise fatorial, obtiveram-se os fatores e os coeficientes (cargas fatoriais) que foram utilizados para estimar os escores fatoriais a partir dos quais calculou-se o IPD.

Os resultados indicam que as quatro variáveis reduziram-se a dois fatores (ver Tabela 1). O primeiro fator apresenta maiores coeficientes de saturação com as variáveis de Degradação da Atividade Animal (DEVANI), Degradação da Mão - de - Obra Rural (DEMORU) e Degradação da Cobertura Vegetal (DECOBV), enquanto o segundo apresenta- se mais correlacionado com a variável Degradação da Cobertura

\footnotetext{
${ }^{5} \mathrm{~A}$ escolha do número de cluster baseou-se no conhecimento que os autores possuem sobre o Estado do Acre, conseqüentemente, tornando assim a análise mais próxima da realidade.
} 
Vegetal (DEVAVE). Estes dois fatores explicam, conjuntamente, 77,05\% da variação total observada nos quatro indicadores iniciais.

Tabela 1 - Cargas fatoriais e coeficientes estimados utilizados para estimar os escores fatoriais após efetuada a rotação ortogonal pelo método Varimax.

\begin{tabular}{llccc}
\hline \multicolumn{1}{c}{ Variáveis } & \multicolumn{2}{c}{$\begin{array}{c}\text { Cargas fatoriais após a } \\
\text { rotação varimax }\end{array}$} & $\begin{array}{c}\text { Coeficientes utilizados para } \\
\text { estimar os escores. }\end{array}$ \\
\hline \hline DEVANI & $\mathbf{0 , 8 8 0 0 0}$ & $8,510 \mathrm{e}-2$ & 0,87500 & $-0,12400$ \\
DEVAVE & $8,593 \mathrm{e}-2$ & $\mathbf{0 , 9 9 3 0 0}$ & 0,32000 & 0,94400 \\
DEMORU & $\mathbf{0 , 8 0 0 0 0}$ & 0,14100 & 0,81100 & $-0,05310$ \\
DECOBV & $\mathbf{0 , 8 0 4 0 0}$ & $-0,00173$ & 0,78100 & $-0,19300$ \\
\hline
\end{tabular}

Fonte: Resultados da pesquisa.

Com base nos indicadores econômicos, biológico e demográfico e utilizando-se os escores fatoriais obtidos com o método de análise fatorial, foi possível estimar o IPD como explicitado na equação (8). Uma vez estimado o IPD (ver Tabela 3) e após encontrar os pesos associados a cada um dos indicadores (ver Tabela 2), os quais foram obtidos por meio de uma análise de regressão linear em que o IPD foi a variável dependente e DEVANI, DEVAVE, DEMORU e DECOBV foram as variáveis independentes, pode-se, então, estimar o ID (ver Tabela 3).

Tabela 2 - Pesos e elasticidades associados ao IPD

\begin{tabular}{lcc}
\hline \multicolumn{1}{c}{ Variáveis } & Pesos & Elasticidades \\
\hline \hline DECOBV & 0,07 & 0,11 \\
DEMORU & 0,13 & 0,12 \\
DEVANI & 0,23 & $\mathbf{0 , 2 5}$ \\
DEVAVE & 0,57 & $\mathbf{0 , 5 4}$ \\
\hline \hline
\end{tabular}

Fonte: Resultados da pesquisa.

Como pode ser visto, as duas variáveis econômicas são as que mais influenciam o IPD e, conseqüentemente, o ID, o que pode ser constatado observando-se os impactos dados pelas elasticidades. Em outros ter- 
mos, pode-se dizer que uma variação de 1\% na variável DEVAVE ocasiona uma variação de aproximadamente $0,54 \%$ no IDP. Por outro lado, uma variação de $1 \%$ em DEVANI ocasiona uma variação de $0,25 \%$ no IPD, sendo estas as elasticidades mais significativas.

Tabela 3 - Indicadores e índice de degradação ambiental

\begin{tabular}{|c|c|c|c|c|c|c|}
\hline \multirow{2}{*}{ Regional/Município } & \multicolumn{4}{|c|}{ INDICADORES } & \multicolumn{2}{|c|}{ ÍNDICE } \\
\hline & DECOBV & DEVAVE & DEVANI & DEMORU & IDP \% & ID \% \\
\hline \multicolumn{7}{|l|}{ Alto Acre } \\
\hline Assis Brasil & 0,00 & 46,47 & 0,00 & 44,37 & 0,43 & 39,17 \\
\hline Brasiléia & 61,92 & 48,58 & 62,34 & 59,10 & 0,69 & 65,95 \\
\hline Epitaciolândia & 57,68 & 18,17 & 54,05 & 70,61 & 0,41 & 44,03 \\
\hline Xapuri & 54,63 & 12,62 & 46,57 & 68,09 & 0,34 & 37,41 \\
\hline \multicolumn{7}{|l|}{ Baixo Acre } \\
\hline Acrelândia & 48,33 & 57,21 & 48,75 & 26,59 & 0,65 & 61,76 \\
\hline Bujari & 18,88 & 28,33 & 31,74 & 86,50 & 0,34 & 43,92 \\
\hline Capixaba & 72,26 & 0,00 & 60,99 & 78,63 & 0,50 & 35,95 \\
\hline Plácido de Castro & 31,96 & 43,23 & 14,87 & 47,28 & 0,40 & 44,36 \\
\hline Porto Acre & 69,46 & 0,00 & 2,29 & 38,93 & 0,13 & 12,80 \\
\hline Rio Branco & 27,62 & 10,22 & 20,66 & 60,12 & 0,15 & 24,82 \\
\hline Senador Guimard & 34,97 & 30,07 & 34,01 & 58,16 & 0,33 & 42,66 \\
\hline \multicolumn{7}{|l|}{ Purus } \\
\hline Manoel Urbano & 0,00 & 0,00 & 0,00 & 11,36 & 0,01 & 1,80 \\
\hline Santa Rosa & 60,20 & 29,19 & 36,70 & 31,90 & 0,31 & 40,82 \\
\hline Sena Madureira & 54,31 & 24,99 & 52,86 & 32,00 & 0,32 & 42,00 \\
\hline \multicolumn{7}{|l|}{ Tarauacá/Envira } \\
\hline Feijó & 65,73 & 12,62 & 0,00 & 0,00 & 0,08 & 14,39 \\
\hline Jordão & 34,92 & 0,00 & 26,65 & 0,00 & 0,06 & 10,56 \\
\hline Tarauacá & 60,12 & 39,15 & 27,56 & 30,08 & 0,37 & 44,83 \\
\hline \multicolumn{7}{|l|}{ Juruá } \\
\hline Cruzeiro do Sul & 0,00 & 0,00 & 0,00 & 0,00 & 0,01 & 0,00 \\
\hline Mâncio Lima & 0,00 & 0,00 & 0,00 & 0,00 & 0,01 & 0,00 \\
\hline Marechal Thaumaturgo & 29,68 & 44,20 & 0,00 & 0,00 & 0,34 & 33,13 \\
\hline Porto Walter & 0,00 & 37,93 & 0,00 & 0,00 & 0,28 & 26,24 \\
\hline Rodrigues Alves & 0,00 & 0,00 & 34,38 & 0,00 & 0,03 & 9,74 \\
\hline Mínimo & 0,00 & 0,00 & - & 0,00 & 0,01 & 0,00 \\
\hline Média & 35,58 & 21,95 & 25,20 & 33,80 & 0,28 & 30,74 \\
\hline Máximo & 72,26 & 57,21 & 62,34 & 86,50 & 0,69 & 65,95 \\
\hline Desvio Padrão & 26,42 & 19,42 & 22,58 & 29,39 & 0,20 & 19,12 \\
\hline
\end{tabular}

Fonte: Resultados da pesquisa. 
Como podem ser visualizados na Tabela 3, os resultados indicam que o Estado do Acre possui um ID médio de 30,74\%, tendo como ID's mínimos os municípios de Cruzeiro do Sul, Mâncio Lima e Manoel Urbano, onde os dois primeiros municípios possuem um ID igual a zero e o terceiro município possui de ID de 1,80\%.

Já a Tabela 4 contém as médias dos indicadores utilizados e do ID para cada regional do Estado do Acre, tendo-se uma visão geral do comportamento dos referidos indicadores de degradação bem como de seu índice.

Tabela 4 - Média dos Indicadores e dos ID's das regionais do Estado do Acre

\begin{tabular}{lcccccc}
\hline \multirow{2}{*}{ REGIONAL } & \multicolumn{4}{c}{ INDICADORES } & \multicolumn{2}{c}{ ÍNDICE } \\
\cline { 2 - 7 } & DECOBV & DEVAVE & DEVANI & DEMORU & IDP & ID \\
\hline \hline Alto Acre & 43,56 & 31,46 & 40,74 & 60,54 & 0,47 & 46,64 \\
Baixo Acre & 43,35 & 24,15 & 30,47 & 56,60 & 0,36 & 38,04 \\
Purus & 38,17 & 18,06 & 29,85 & 25,08 & 0,21 & 28,21 \\
Tarauacá/Envira & 53,59 & 17,26 & 18,07 & 10,03 & 0,17 & 23,26 \\
Juruá & 5,94 & 16,43 & 6,88 & - & 0,13 & 13,82 \\
\hline \hline
\end{tabular}

Fonte: Resultados da pesquisa.

Conforme esta Tabela, observa-se que os maiores indicadores e índices de degradação ambiental encontram-se nas regionais do Alto e Baixo Acre. Tal resultado pode ser justificado pelo fato de estas regionais possuírem a maior quantidade de projetos de colonização, assentamentos e atividades agroextrativistas em relação às demais, uma vez que detém, conjuntamente, $54,83 \%$ das áreas do Estado destinadas a esta finalidade. Com relação à capacidade de assentamento familiar, estas duas regionais representam $61,32 \%$ da capacidade total destes projetos. GOVERNO DO ESTADO DO ACRE (2000).

De acordo com os resultados do relatório final do ZEE (2000), as regionais do Alto e Baixo Acre apresentam maiores índices de desflorestamento e queimadas em virtude do maior ponto de concentração dos projetos de assentamentos, grandes fazendas, estradas e centros urbanos. 
Como forma de verificar características semelhantes entre municípios foi realizada uma análise de cluster. O número de clusters foi definido de forma que os agrupamentos apresentassem um alto grau de homogeneidade entre os municípios e um al to grau de heterogeneidade entre os clusters. Os clusters foram formados tendo como variáveis de agrupamento os indicadores de degradação ambiental.

Foram definidos 5 clusters, conforme resultados da Tabela 5.

Tabela 5 - Clusters ambientais dos municípios do Acre

\begin{tabular}{|c|c|c|c|c|c|c|}
\hline \multirow{2}{*}{ Clusters } & \multicolumn{4}{|c|}{ INDICADORES } & \multicolumn{2}{|c|}{ INDICES } \\
\hline & DECOBV & DEVAVE & DEVANI & DEMORU & IPD-1 & ID-1 \\
\hline \multicolumn{7}{|l|}{ Cluster - 1} \\
\hline Cruzeiro do Sul & - & - & - & - & 0,01 & - \\
\hline Jordão & 34,92 & - & 26,65 & - & 0,06 & 10,56 \\
\hline Mâncio Lima & - & - & - & - & 0,01 & - \\
\hline Manoel Urbano & - & - & - & 11,36 & 0,01 & 1,80 \\
\hline Rodrigues Alves & - & - & 34,38 & - & 0,03 & 9,74 \\
\hline Máximo & 34,92 & - & 34,38 & 11,36 & 0,06 & 10,56 \\
\hline Média & 6,98 & - & 12,21 & 2,27 & 0,02 & 4,42 \\
\hline Mínimo & - & - & - & - & 0,01 & - \\
\hline Desvio-Padrão & 15,62 & - & 16,94 & 5,08 & 0,02 & 5,29 \\
\hline \multicolumn{7}{|l|}{ Cluster - 2} \\
\hline Acrelândia & 48,33 & 57,21 & 48,75 & 26,59 & 0,65 & 61,76 \\
\hline Assis Brasil & - & 46,47 & - & 44,37 & 0,43 & 39,17 \\
\hline Mar. Thaumaturgo & 29,68 & 44,20 & - & - & 0,34 & 33,13 \\
\hline Plácido de Castro & 31,96 & 43,23 & 14,87 & 47,28 & 0,40 & 44,36 \\
\hline Porto Walter & - & 37,93 & - & - & 0,28 & 26,24 \\
\hline Máximo & 48,33 & 57,21 & 48,75 & 47,28 & 0,65 & 61,76 \\
\hline Média & 21,99 & 45,81 & 12,72 & 23,65 & 0,42 & 40,93 \\
\hline Mínimo & - & 37,93 & - & - & 0,28 & 26,24 \\
\hline Desvio-Padrão & 21,33 & 7,10 & 21,14 & 22,99 & 0,14 & 13,47 \\
\hline \multicolumn{7}{|l|}{ Cluster - 3} \\
\hline Feijó & 65,73 & 12,62 & - & - & 0,08 & 14,39 \\
\hline Porto Acre & 69,46 & - & 2,29 & 38,93 & 0,13 & 12,80 \\
\hline S.Rosa do Purus & 60,20 & 29,19 & 36,70 & 31,90 & 0,31 & 40,82 \\
\hline Máximo & 69,46 & 29,19 & 36,70 & 38,93 & 0,31 & 40,82 \\
\hline Média & 65,13 & 13,94 & 13,00 & 23,61 & 0,17 & 22,67 \\
\hline Mínimo & 60,20 & - & - & - & 0,08 & 12,80 \\
\hline Desvio-Padrão & 4,66 & 14,64 & 20,56 & 20,74 & 0,12 & 15,74 \\
\hline
\end{tabular}




\begin{tabular}{lrrrrrr} 
Cluster -4 & & & & & & \\
$\quad$ Bujari & 18,88 & 28,33 & 31,74 & 86,50 & 0,34 & 43,92 \\
$\quad$ Rio Branco & 27,62 & 10,22 & 20,66 & 60,12 & 0,15 & 24,82 \\
$\quad$ Senador Guimard & 34,97 & 30,07 & 34,01 & 58,16 & 0,33 & 42,66 \\
Máximo & $\mathbf{3 4 , 9 7}$ & $\mathbf{3 0 , 0 7}$ & $\mathbf{3 4 , 0 1}$ & $\mathbf{8 6 , 5 0}$ & $\mathbf{0 , 3 4}$ & $\mathbf{4 3 , 9 2}$ \\
Média & $\mathbf{2 7 , 1 5}$ & $\mathbf{2 2 , 8 8}$ & $\mathbf{2 8 , 8 0}$ & $\mathbf{6 8 , 2 6}$ & $\mathbf{0 , 2 7}$ & $\mathbf{3 7 , 1 3}$ \\
Mínimo & $\mathbf{1 8 , 8 8}$ & $\mathbf{1 0 , 2 2}$ & $\mathbf{2 0 , 6 6}$ & $\mathbf{5 8 , 1 6}$ & $\mathbf{0 , 1 5}$ & $\mathbf{2 4 , 8 2}$ \\
Desvio-Padrão & $\mathbf{8 , 0 6}$ & $\mathbf{1 0 , 9 9}$ & $\mathbf{7 , 1 4}$ & $\mathbf{1 5 , 8 3}$ & $\mathbf{0 , 1 0}$ & $\mathbf{1 0 , 6 8}$ \\
Cluster - 5 & & & & & & \\
$\quad$ Brasiléia & 61,92 & 48,58 & 62,34 & 59,10 & 0,69 & 65,95 \\
$\quad$ Capixaba & 72,26 & - & 60,99 & 78,63 & 0,50 & 35,95 \\
$\quad$ Epitaciolândia & 57,68 & 18,17 & 54,05 & 70,61 & 0,41 & 44,03 \\
$\quad$ Sena Madureira & 54,31 & 24,99 & 52,86 & 32,00 & 0,32 & 42,00 \\
$\quad$ Xapuri & 54,63 & 12,62 & 46,57 & 68,09 & 0,34 & 37,41 \\
Máximo & $\mathbf{7 2 , 2 6}$ & $\mathbf{4 8 , 5 8}$ & $\mathbf{6 2 , 3 4}$ & $\mathbf{7 8 , 6 3}$ & $\mathbf{0 , 6 9}$ & $\mathbf{6 5 , 9 5}$ \\
Média & $\mathbf{6 0 , 1 6}$ & $\mathbf{2 0 , 8 7}$ & $\mathbf{5 5 , 3 6}$ & $\mathbf{6 1 , 6 8}$ & $\mathbf{0 , 4 5}$ & $\mathbf{4 5 , 0 7}$ \\
Mínimo & $\mathbf{5 4 , 3 1}$ & - & $\mathbf{4 6 , 5 7}$ & $\mathbf{3 2 , 0 0}$ & $\mathbf{0 , 3 2}$ & $\mathbf{3 5 , 9 5}$ \\
Desvio-Padrão & $\mathbf{7 , 4 2}$ & $\mathbf{1 8 , 0 0}$ & $\mathbf{6 , 4 3}$ & $\mathbf{1 8 , 0 0}$ & $\mathbf{0 , 1 5}$ & $\mathbf{1 2 , 1 3}$ \\
\hline
\end{tabular}

Fonte: Resultados da pesquisa

O cluster 1 possui cinco municípios, dos quais três pertencem à regional do Juruá, um pertence à regional do Purus e um à regional Tarauacá/ Envira. A principal característica deste agrupamento é a sua baixa média nos indicadores e índices de degradação ambiental, principalmente, nos indicadores DEVAVE e DEMORU.

0 cluster 2, também possui 5 municípios, dos quais dois pertencentes à regional do Baixo Acre, dois do Juruá e um da regional do Alto Acre. A principal característica deste agrupamento está associada ao indicador DEVAVE, significando que neste cluster existe uma baixa produtividade vegetal.

O cluster 3 possui três municípios, cada um deles pertencentes às regionais de Tarauacá/Envira, Baixo Acre e Purus. O indicador DECOBV, neste agrupamento, foi a variável que mais contribuiu para mostrar a degradação do meio ambiente.

O cluster 4 possui três municípios, todos pertencentes à regional do Baixo Acre. $O$ indicador de degradação ambiental que mais se destacou neste cluster foi DEMORU, dado que estes municípios comportam mais de $50 \%$ da população do Estado do Acre. 
Por último, o cluster 5, é composto por cinco municípios, dos quais três são da regional do Alto Acre, um da regional do Purus e um da regional do Baixo Acre. Este último agrupamento possui três indicadores de degradação ambiental com valores bastante elevados, sendo que os indicadores DECOBV, DEVANI e DEMORU apresentam valores preocupantes.

Diante do exposto, pode-se observar uma convergência entre os resultados encontrados neste trabal ho e aqueles obtidos no ZEE (2000). No entanto, é mister esclarecer que o ID é um índice composto por quatro indicadores e, em alguns casos, existe uma diferença significativa entre o ID e o indicador presente no ZEE (2000) calculado pela FUNTAC (Fundação de Tecnologia do Estado do Acre). Esta diferença foi ocasionada pela incorporação de aspectos econômicos e populacionais no ID, o que não acontece com o indicador da FUNTAC.

\section{5 - Conclusão}

O objetivo principal deste trabalho foi elaborar um Índice de Degradação (ID) que fosse capaz de aferir o atual estágio de degradação ambiental em que se encontram os 22 municípios do Estado do Acre, na Amazônia Ocidental Brasileira, procurando, também, agrupá-los em clusters para verificar características semelhantes entre eles.

Como visto, os resultados indicam que o Estado do Acre possui um ID médio de $30,74 \%$, tendo como ID's mínimos os municípios de Cruzeiro do Sul, Mâncio Lima e Manoel Urbano, onde os dois primeiros municípios possuem um ID igual a zero e o terceiro município possui ID de $1,80 \%$.

No que diz respeito às regionais, observou-se que as regionais do Baixo e Alto Acre apresentaram as maiores médias do ID, em contrapartida, a regional do Juruá apresentou um baixo ID, possuindo como destaque os municípios de Cruzeiro do Sul e Rodrigues Alves.

A análise de cluster mostrou que determinadas regiões possuem um bom estado de conservação ambiental, no entanto, também deixou claro que alguns indicadores de degradação apresentam valores extremamente elevados. 0 cluster 5 possui médias altíssimas dos indicadores de degradação bem como no índice de degradação. Contrariamen- 
te, o cluster 1 apresenta uma situação confortável em termos de degradação do meio ambiente.

Outro aspecto importante a ser ressaltado foi a convergência entre os resultados encontrados neste trabalho e aqueles obtidos no ZEE (2000). No entanto, como visto, em alguns casos, existe uma diferença significativa entre o ID e o indicador presente no ZEE (2000), diferença esta ocasionada pela incorporação de aspectos econômicos e populacionais no ID, o que não acontece com o indicador da FUNTAC.

A utilização de outros indicadores para avaliar a degradação ambiental contribui de forma significativa para outros estudos. Uma das limitações deste trabalho reside na análise de apenas quatro indicadores. Outra limitação reside nas condições de estabilidade propostas pela expressão 12.

Conclusivamente, pode-se dizer que, apesar de apresentar índices de degradação relativamente baixos em alguns casos, isto não significa que a situação do Acre seja cômoda, mas, pelo contrário, é muito preocupante, dado que, por ser um Estado relativamente novo e pouco desenvolvido, alguns municípios possuem um ID que ultrapassou o valor de $60 \%$.

Portanto, fica evidente que o processo de desenvolvimento do Estado do Acre que se concentrou nas regionais do Baixo e Alto Acre, trouxe grandes danos ambientais ao Estado.

\section{6 - Referência Bibliográfica}

BASILEVSKY, A Statistical Factor Analysis and Related Methods: Theory and Applications, New York, 1994.

DILLON, W. e GOLDSTEIN, M. Multivariate Analysis: Methods and Applications, New York, 1984.

FERNANDES, T. A. G e LIM A, J. E. Uso de Análise Multivariada para Identificação de Sistemas de Produção. Brasília: Revista Pesquisa Agropecuária Brasileira, 26(10): 1.823-1.836, out. 1991.

FERNAU, M.E.; SAMSON, P.J. Use of cluster analysis to define periods of similar meteorology and precipitation hemistry in Eastern North America. Partl: Transport patterns. J ournal of Applied Meteorology, Michigan, v.29, p.735-761, 1990. 
GONG, X.; RICHMAN, M.B. On the application to growing season precipitation data in North America East of the rockies. J ournal of Climate, Oklahoma, v.8, p.897-931, 1995.

GOVERNO DO ESTADO DO ACRE. Zoneamento Ecológico-Econômico. Acre: SECTMA, V. I, II e III, 2000.

IBGE - Instituto Brasileiro de Geografia e Estatística. Censo Agropecuário. n.3, Rio de Janeiro: IBGE, 1995.

JOHNSON, A e WICHERN, D. Applied Multivariate Statistical Analysis, New Jersey, 1988.

LEMOS, J.J.S. Desertification of Drylands in Northeast of Brazil, Riverside, Califórnia. Economic Department da University of California, 1995.

LEM OS, J.J.S. Indicadores de Degradação no N ordeste Sub-úmido e Semi-árido. Revista SOBER, 2000, p.1-10.

POLLAK, L.M.; CORBETT, J.D. Using GIS datasets to classify maize-growing regions in M exico and Central America. Agronomy J ournal, v.85, p.11331139, 1993.

Recebido em março de 2003 e revisto em março de 2004. 